How to cite this article:

Lee, T. S., \& Sulaiman, R. (2019). Preliminary analysis of wireless collaborative network on mobile devices. Journal of Information and Communication Technology, 18(3), 327343.

\title{
PRELIMINARY ANALYSIS OF WIRELESS COLLABORATIVE NETWORK ON MOBILE DEVICES
}

\author{
Thian Seng Lee \& Riza Sulaiman \\ Institute of Visual Informatics, Universiti Kebangsaan Malaysia, \\ Malaysia
}

thianseng01@yahoo.com; riza@ukm.edu.my

\begin{abstract}
Researchers and companies of the telecommunication sector have been working hard to enhance wireless network infrastructure to fulfill end users' needs for Internet connectivity. However, it is not easy to satisfy the high demands and expectations of end users always. There are some situations where Internet connectivity is not enabled due to location and environmental constraints. For example, network infrastructure breakdown during a disaster or the user's device is out of a network coverage area. Besides relying only on network infrastructure provided by telecommunication companies, one possible strategy to provide Internet connectivity in the ubiquitous environment is to exploit the functionality of end-user mobile devices as a tool to share Internet connectivity. This paper aims to identify the constraints and challenges of setting up Wireless Collaborative Network (WCN) using end user devices. To identify the constraints and challenges, the researchers reviewed and categorized past and current research focusing on collaborating mobile devices in the mobile ad-hoc network for communication and resource sharing. For validation, a simple test case was conducted which involved
\end{abstract}


a few mobile devices in a random formation. Based on the findings, it was concluded that the biggest challenge of setting up WCN was the ability of mobile devices to provide bridging functionality. This paper also discussed the expected architecture of WCN and suggests simulation tools with relevant metrics to be considered in this research area.

Keywords: Internet connectivity sharing, mobile ad-hoc network, wireless collaborative network, Wi-Fi.

\section{INTRODUCTION}

The rapid advancement of new technology has brought a huge impact to the mobile device industry. Mobile devices are getting more powerful, with more functionality, higher processing power, and larger capacity. Based on a survey reported by Accenture (2012), "mobile Internet usage continues to be on a sharp upward trajectory." Other than personal computers, mobile devices are the primary medium to access the Internet across different groups of people. Based on mobile behaviour report, the activities performed by most of mobile device owners are emailing, text messaging, searching information on the Internet, logging to social networking as well as watching videos or movies (ExactTarget, 2014). Based on a study of consumers' smartphone usage behaviour in Malaysia, Osman et al. (2012) reported that Malaysian consumers have a high usage of the smartphone as the medium for Internet access for daily activities such as blogging, checking emails and entertainment. In conclusion, based on all the reports studied, Internet access is essential for most mobile device users.

Unfortunately, there are some situations where Internet connectivity is not enabled due to location and environmental constraints. For example, a mobile data plan subscriber just needs a few bytes to send an important email urgently, but the Internet access quota is used up and the connectivity becomes very slow or even worse, zero connectivity. Wi-Fi access point then turns out to be the user's alternate solution. Somehow, there is no guarantee for mobile users to have open access hotspots available nearby. Other than usage on a daily basis, the Internet or any wireless network connectivity could also be used for critical situations such as during disasters. In the case of communication breakdown during a disaster, a temporary mobile ad-hoc network infrastructure could be used in search and rescue work. A Wireless Collaborative Network (WCN) with full connectivity over far distances could be established through the social collaboration of mobile devices which is already available ubiquitously. The 
researchers aimed to identify the challenges and constraints in establishing WCN. Constructing a WCN could cover a wide research field from physical hardware to computing software. This study focuses on the scope of exploiting the functionality and utilization of end-user mobile devices.

\section{LITERATURE REVIEW}

Numerous researches have been conducted to explore and design different approaches to maintain wireless network connectivity. Researchers had developed proposals, designs, software, framework, architecture, and protocol to support connectivity. Some even explored the possibility of using robots or inventing devices to act as a bridge or gateway to maintain wireless network connectivity. In general, the focus of the research area can be categorized as either hardware based or software based. Both categories have the same objective of providing continuous wireless network connectivity for mobile devices. In the next section, we present related work in these two categories.

\section{Hardware-centric Wireless Collaborative Network}

This section discusses the work done in integrating networking hardware for wireless network connectivity. The hardware covers the invention of network devices, communication towers, and robots. Stevens et al. (2014) designed a prototype of a portable telecommunication tower. The tower design was claimed to be at a low cost, portable and openly available for replication. Adeyeye and Gardner-Stephen and Palaniswany (2011) presented the communication infrastructure design using a device invented which was known as mesh potato. From the inspiration of cross-field research, some researchers used robots to broaden and optimize wireless network connectivity. Reich et al. (2008) introduced in a research project report, the prototype of a mobile network named Spreadable Connected Autonomic Networks (SCAN) in which any robot with integrated SCAN algorithm will automatically move to maintain connectivity and at the same time works as a wireless access point. Liemhetcharat and Veloso (2012) deployed a team of mobile robots to automatically locate and then tether to an autonomous agent. The robots shared connectivity information using the distributed algorithm developed. Other researchers who utilized robotic technology to enhance wireless network connectivity are Ocana et al. (2005) and Sit et al. (2007). Although some of the works reviewed mainly focused on expanding the robot's wireless network connectivity for localization purposes instead of wireless network connectivity expansion, these concepts and ideas remain relevant. 
Based on the literature, the considerations of hardware integration to wireless network connectivity basically include the total set-up cost, radio interference, the scalability, and the geographical location of the set-up base. Some researchers claimed that the designed prototype is not expensive and is deployable in a real environment (Adeyeye \& Gardner-Stephen, 2011; Liemhetcharat \& Veloso, 2012; Stevens et al., 2014). Despite many successful cases from the literature, where the invented device has proven to work, the researchers still believe that it is not the best solution after considering the cost, complications of setting up the hardware, setting up and extending the wireless network, acceptance by the end user and obtaining approval by the local authorities in the hardware set-up. Comparing this with the common and easily available portable devices such as the mobile phone or tablet which could provide similar functionality, the latter is much cheaper and is already freely available almost everywhere. Therefore, this research concentrated on utilizing the end users' mobile devices. The technique and idea implemented in the hardware invented base in the literature mentioned could be adapted into the researchers' work. For instance, routing protocol, algorithm of scanning and discovery, encryption technique of communication sessions and data transfer, including resource and power management techniques.

\section{Software-centric Wireless Collaborative Network}

This section discusses the work done on designing a software system for wireless network connectivity. The term software system described here includes algorithm, application, protocol, framework, and architecture. Many works have been conducted on creating continuous wireless network connectivity through incorporating software systems into mobile devices. Some researchers have classified the research area as software-defined networking. Nungu et al. (2011) discussed the stages and planning taken to establish local broadband communication networks on a community level. They also reported the challenges in setting up a broadband network which includes financial support, suitable network infrastructure, and equipment with relevant functionality. Based on the report, the researchers have extracted some information about the constraints and challenges of setting up WCN which is needed as part of the researchers' work.

Evidence have shown that the mobility nature of mobile devices can potentially increase the capacity of a wireless network (Grossglauser \& Tse, 2002). Honda and Ikeda (2013) found that the location of the Access Point (AP) will affect the Wi-Fi connection's Quality of Service (QoS). The position of the AP must be manually adjusted with the help of simulation tools (Qualnet) for calculating a suitable location. This method is considered as time-consuming, 
albeit the results showed that Wi-Fi QoS has been improved. In order to assist in the planning of access point or gateway set-up, researchers have developed algorithms and techniques used for the location planning of gateways and routers and for the optimization of routing and channel allocation (Pries et al., 2010; Vanhatupa et al., 2007). Based on the literature on AP positioning, an assumption can be drawn where the real-time location and position of the end user's mobile device in randomized nature will affect the QoS of the entire WCN. The end user's mobile device should have an intelligent mechanism to determine the best available surrounding resources in real time.

Bellavista and Giannelli(2009)introduced the idea ofimplementing middleware in mobile devices to provide multi-hop multi-path Internet connectivity. They achieved the connectivity by exploiting the JSR-82 Bluetooth API and integrating the API into a proposed architecture known as MMHC. The new architecture has been tested and demonstrated success in setting up MultiHop Multi-Path connectivity via Bluetooth interface. However, the research only focused on Bluetooth interface and not much information about Wi-Fi interface is available. Furthermore, it has been observed that middleware works only partially in certain operating system platforms (e.g. MS Windows) and therefore this is contradictory to the main objective as middleware should support connectivity for heterogeneous multi-hop clients. This could be due to the restrictions imposed by the networking hardware manufacturer. In continuing with their previous work, Bellavista et al. (2010) then developed an enhanced middleware which supported Wi-Fi interfaces. The prototype RAMP has been tested and has shown success in setting up a spontaneous network connection to perform data transfers. However, it should be noted that the test case used for the work involved only up to three hops. More hops and devices should be included to draw a more solid and convincing finding. In implementing the concept of mesh protocol network, a mobile application known as Serval Mesh has been developed with the intention of enabling people to stay connected during a crisis such as disasters (Gardner-Stephen et al., 2013). The Serval Mesh mobile application has been published in the Android market and it has proven to be practicable after several field tests were conducted. Based on the literature, the off-the-shelf mobile devices in the mobile network stand a high chance of being utilized to create multi-hop Internet network.

\section{CONSTRAINTS AND CHALLENGES IN SETTING UP WIRELESS COLLABORATIVE NETWORKS}

It is important to note that the constraints and challenges were identified in the scope of the functionality of end user mobile devices. Yet, the researchers 
fervently believe that end users' mobile devices play an important role in setting up the WCN, which is currently underutilized. Six major contributing factors which make up the constraints and challenges of setting up WCN are listed in Table 1 as follows:

Table 1

Contributing factors in the constraints and challenges of setting up WCN

\begin{tabular}{ll}
\hline Factor & Contributing factor and explanation \\
\hline Factor 1 & $\begin{array}{l}\text { Spontaneous resources - The choice of the best available device with } \\
\text { resources at any time is uncertain. }\end{array}$
\end{tabular}

Factor 2 User attitude - The willingness of mobile device users to allow their devices to be part of the $\mathrm{WCN}$ is uncertain.

Factor 3 Network connectivity preservation - Maintaining connectivity between devices is difficult as the devices' movements or direction is beyond control.

Factor 4 Device's interface response time - How fast can the Wi-Fi interface respond? For instance, scanning and connecting to other devices so that the user experience will not be affected by latency.

Factor $5 \quad$ Data security and user privacy - Security and privacy considerations for data sharing in a social sharing environment.

Factor 6 Energy conservation - Battery consumption will increase as workload of the device increases.

\section{CONTRIBUTING FACTORS IN VALIDATION}

A preliminary testing was conducted to validate the contributing factors identified and listed in Table 1. This involved a few mobile devices to set up a simple wireless network. The mobile devices were used to simulate a sample file transfer process. The process was analysed, and observations were recorded to reflect the contributing factors identified in Table 1.

\section{Experimental Set-up for Preliminary Testing}

In this work, the category of mobile devices includes the smartphone, tablet, and phablet because these are potentially powerful and useful sources of connection available ubiquitously. We have chosen Android as the platform 
for testing and prototype development purposes considering its open source platform. We prepared four smartphones and two tablets of different brands running Android operating systems. Figure 5 depicts the set-up of our testing model. P1-P4 represents the smartphones and T1-T2, the tablets used. P1 and P2; T1 and P3; P4 and T2 were interconnected via Bluetooth interface. P2 and $\mathrm{T} 1, \mathrm{P} 3$ and $\mathrm{P} 4$ were interconnected via Wi-Fi interface.

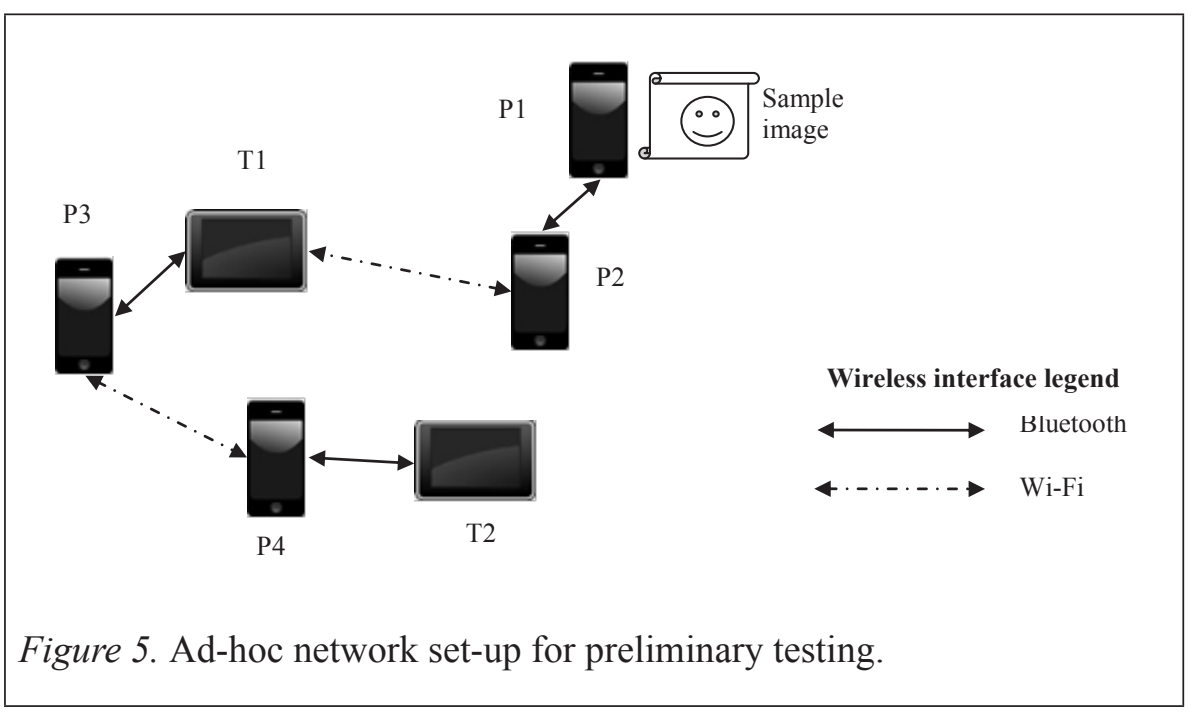

\section{File Transfer Observation}

A random image size of $2 \mathrm{mb}$ was used as the resource to be shared by all devices. The preliminary testing objective was to test if a file can be sent from a source device to all other five devices via the ad-hoc network set-up in parallel with the existing available wireless interfaces. All the mobile devices had at least one or three types of radio (e.g. multi-band cellular, Bluetooth, and Wi-Fi) that can be used for communication purposes with other devices. For the preliminary testing, we began by exploring the higher level of the device system to inspect if there was any possible way of integrating the devices as part of WCN with minimal coding or modifications to the existing system of the devices. As the multi-band cellular interface had been hardcoded by the manufacturer, it was almost impossible for us to modify and make use of the interface. We had only Bluetooth and Wi-Fi interface as the options to set up the WCN. Furthermore, there was to some extent certain flexibility and functionality which could be controlled or programmed by the user. For Bluetooth connections, the file-sharing operation was done manually via the Bluetooth file sharing feature in the Android system. For Wi-Fi connections, the application named SHAREit from Google Play was installed on every device and used for file sharing. 


\section{Findings}

While performing the file transfer operations from P1 to T2 (as in Figure 1) we observed the activity and analysed the process to validate the contributing factors as identified in Table 1. Table 2 summarises the premise and observation outcomes which reflect the contributing factors of Table 1.

\section{Table 2}

\section{Explanation on the validation of the identified contributing factors}

\begin{tabular}{|c|c|c|}
\hline Contributing Factor & Validation by Observation & Premise \\
\hline Spontaneous Resources & $\begin{array}{l}\text { Device } \mathrm{P} 2 \text { is located near } \\
\text { to device } \mathrm{T} 2 \text { but due to } \\
\text { the limited connection } \\
\text { interface of } \mathrm{P} 2 \text { which } \\
\text { has connected to } \mathrm{P} 1 \text { and } \\
\mathrm{T} 1 \text {, the file has to pass } \\
\text { through } \mathrm{T} 1, \mathrm{P} 3 \text {, and } \mathrm{P} 4 \text {. }\end{array}$ & $\begin{array}{l}\text { This shows that an } \\
\text { intelligent technique is } \\
\text { needed for a device to } \\
\text { detect the best and nearest } \\
\text { available resources. }\end{array}$ \\
\hline User Attitude & $\begin{array}{l}\text { We have full control on } \\
\text { file distribution. However, } \\
\text { we could not control the } \\
\text { respected device's owner } \\
\text { in real time. }\end{array}$ & $\begin{array}{l}\text { This shows that a unified } \\
\text { technique is needed to } \\
\text { encourage collaboration } \\
\text { among users. }\end{array}$ \\
\hline $\begin{array}{l}\text { Network Connectivity } \\
\text { Preservation } \\
\text { - Maintaining connectivity } \\
\text { between devices is difficult as } \\
\text { the movements/direction of the } \\
\text { devices is beyond control. }\end{array}$ & $\begin{array}{l}\text { This was observed when } \\
\text { a file sending session } \\
\text { between any paired } \\
\text { devices failed due to any } \\
\text { one of the devices moving } \\
\text { away and eventually out } \\
\text { of the connection range } \\
\text { before the sending process } \\
\text { ended. }\end{array}$ & $\begin{array}{l}\text { This shows that a } \\
\text { technique is needed } \\
\text { for the device to stay } \\
\text { connected to the } \\
\text { neighbour node whenever } \\
\text { possible. }\end{array}$ \\
\hline $\begin{array}{l}\text { Device's Interface Response } \\
\text { Time } \\
\text { - How fast can the Wi-Fi } \\
\text { interface respond? For instance, } \\
\text { scanning and connecting to } \\
\text { other devices so that the user } \\
\text { experience will not be affected } \\
\text { by latency. }\end{array}$ & $\begin{array}{l}\text { BT and Wi-Fi were } \\
\text { compared. BT operates } \\
\text { in shorter and consistent } \\
\text { connection time. } \\
\text { Wi-Fi connection } \\
\text { takes longer with } \\
\text { inconsistent timing (up } \\
\text { to } 5 \text { seconds of variance) } \\
\text { when connecting to } \\
\text { neighbouring devices. }\end{array}$ & $\begin{array}{l}\text { This shows that a rapid } \\
\text { technique is needed for } \\
\text { the device to perform as } \\
\text { soon as the connection } \\
\text { operation is needed. }\end{array}$ \\
\hline
\end{tabular}




\begin{tabular}{|c|c|c|}
\hline Contributing Factor & Validation by Observation & Premise \\
\hline $\begin{array}{l}\text { Data Security and User Privacy } \\
\text { - Security and privacy } \\
\text { considerations for data sharing } \\
\text { in a social sharing environment. }\end{array}$ & $\begin{array}{l}\text { This was clearly observed } \\
\text { as the file can be seen by } \\
\text { all devices involved in the } \\
\text { transfer process. }\end{array}$ & $\begin{array}{l}\text { This shows that a secure } \\
\text { technique is needed to } \\
\text { ensure that data flow } \\
\text { between networks are } \\
\text { encrypted and only } \\
\text { available to the final } \\
\text { receiver. }\end{array}$ \\
\hline $\begin{array}{l}\text { Energy Conservation } \\
\text { - Battery consumption will } \\
\text { increase as the workload of the } \\
\text { device increases. }\end{array}$ & $\begin{array}{l}\text { All devices were fully } \\
\text { charged and the file } \\
\text { transfer process from } \\
\text { P1 to T2 was performed } \\
\text { for five times. After the } \\
\text { fifth time of the transfer } \\
\text { process, the battery level } \\
\text { (\% under Settings }> \\
\text { Battery menu) for devices, } \\
\text { P2, T1, P3 and P4 were } \\
(2-3 \%) \text { lower than the } \\
\text { devices, P1 and T2. }\end{array}$ & $\begin{array}{l}\text { This shows that an } \\
\text { efficient technique is } \\
\text { needed to minimize the } \\
\text { power consumption of the } \\
\text { device. }\end{array}$ \\
\hline
\end{tabular}

Based on the testing conducted, it was confirmed that it is possible to partially set up the WCN with resource sharing facilities by using two different wireless interfaces of a mobile device. To construct a WCN, the network topology should be in the form of Mesh network which is not possible with the default wireless interface of the mobile devices. In terms of performance, the inconsistency of data transfer speeds and the connectivity range were the major limitations. The Wi-Fi interface supports a wider range of up to 46 metres indoor and 92 metres outdoor; with a data transfer rate about 54 megabits per second. It is higher than Bluetooth which has a data transfer rate of about 3 megabits per second and an approximate range of 10 metres. Hence, a file can be sent further and faster in the WCN via the Wi-Fi interface. This research was targeted at a wider range of resources and connectivity sharing in WCN; therefore the Wi-Fi interface is chosen as the focus in future work.

The AWT program was created by the Android developer to allow wireless tethering or ad-hoc access point by a mobile device to share cellular data service with other mobile devices via Wi-Fi interface. A few researchers have tried to construct WCN using AWT (Gardner-Stephen \& Palaniswamy, 2011; Han et al., 2014). A mobile device with AWT program running can support parallel connections with only a limited number of other mobile devices at a time. On top of that, the biggest limitation is that the Wi-Fi interface of a device cannot be used for another connection when the interface is in use (Gardner-Stephen \& Palaniswamy, 2011; Liu et al., 2016; Wang et al., 2016). 
Figure 2 demonstrates a scenario wherein mobile device A, which is connected to an access point through Wi-Fi interface, will not be able to become a hotspot for other devices, say, mobile device B that is nearby mobile device A. That is the main reason why some researchers suggested the integration of multiple network interfaces of mobile devices in order to set up the WCN (Liu et al., 2016; Wang et al., 2016). Even if this can be solved using additional Wi-Fi component hardware, it is not practical and not possible for some mobile devices currently available in the market. Although there are studies and proposed solutions for the problems discussed, yet there is none at this point in time which is fully established and realistically applicable in the mobile network environment.

Based on our findings, it has been confirmed that to establish the WCN with WiFi interface, only the functionality of a mobile device to become the connectivity bridge for two mobile devices via Wi-Fi interface is needed as shown in Figure 3.
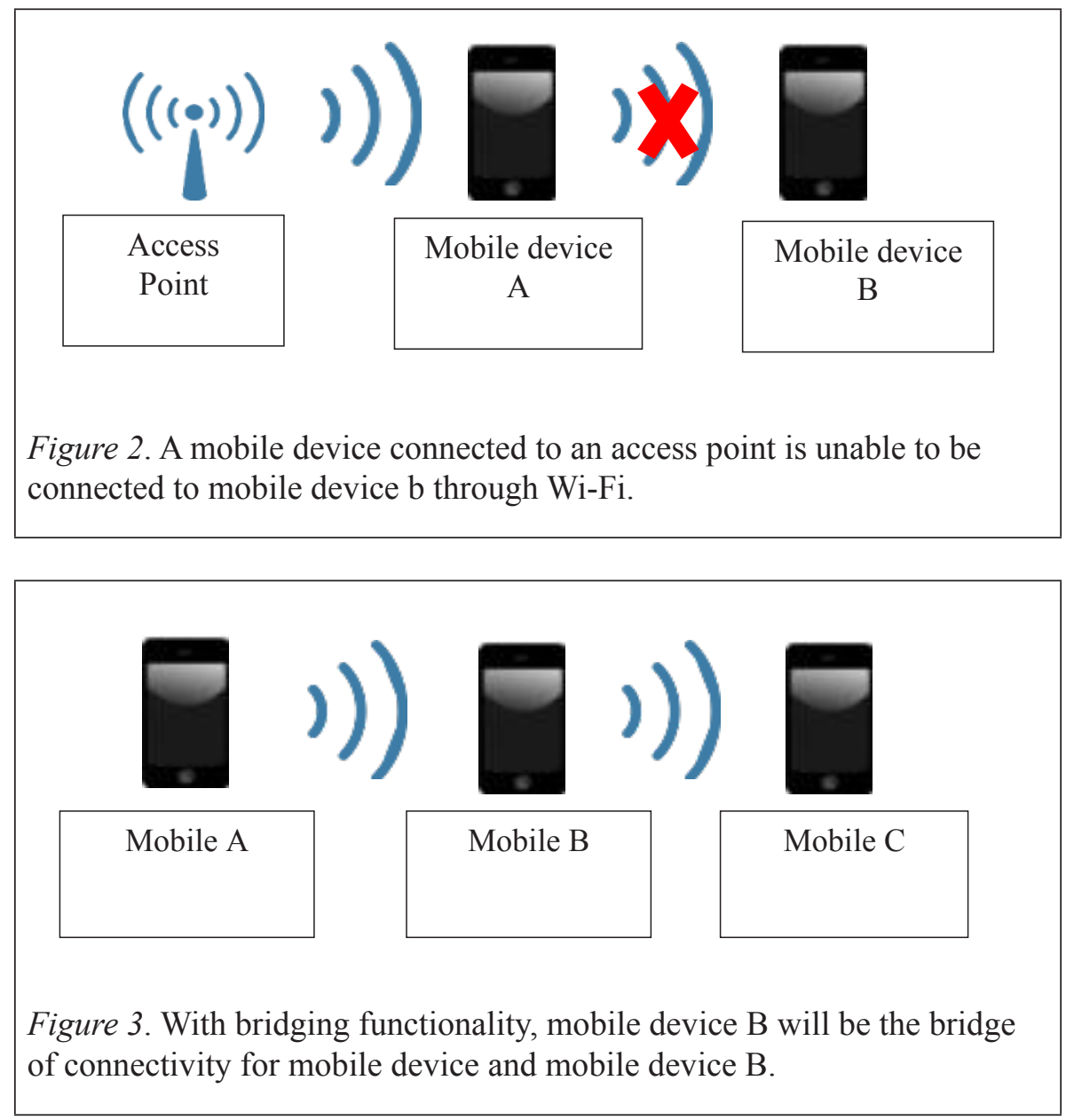


\section{DISCUSSION}

Bellavista (2014) suggested that mobile devices inter-network connectivity at all times is achievable by optimizing the potential of the networking functionalities of underutilized mobile devices. There is a need for an innovative service to set up a space on social collaboration so that mobile users can share information and resources in the ubiquitous environment. The information shared among mobile devices could be the location of the nearest free WiFi access point or information of diverted wireless network connections via the social collaboration of mobile devices. Researchers have been exploiting the functionality of targeted mobile devices to serve as tools of WCN due to the rising trend of using mobile devices such as the smartphone, tablet or phablet (Bellavista, 2014; Bellavista \& Giannelli, 2009; Gardner-Stephen \& Palaniswamy, 2011; Han et al., 2014). To establish a WCN, a mobile device should be able to connect at least two other devices together.

In the effort to enable resource sharing between mobile devices, the ad-hoc mode through Wi-Fi interface has been introduced to allow connectivity between two or more mobile devices. But the interface introduced has limited functionality and applicable resources sharing in a small area network. Wi-Fi Direct protocol has been introduced as a progression of Wi-Fi ad-hoc mode, with the aim of being the standardized platform for file sharing or two-way communication functionalities for mobile devices. The new protocol introduced was aimed at enhancing stability and improving the ease of use for wireless resource sharing. Unfortunately, it is not fully supported and available on all devices as some devices are only able to perform one-to-one connection, and this has been reported by Liu et al. (2016). Nevertheless, social collaboration for mobile devices can be achieved by cautiously planning and enhancing the ability of mobile devices as the main player in the WCN (Adeyeye \& GardnerStephen, 2011; Camp \& Knightly, 2008; Wang et al., 2016). The essential rule of thumb is that the device needs to be connected and at the same time connected to other devices.

In our long-term research project, we intend to explore the possibility of utilizing mobile devices as the medium for setting up a WCN with full connectivity. It is hoped that the information shared in this paper will help new researchers to address the constraints and challenges of setting up WCN quickly and easily so that less time is needed to identify the problem statement. It is important to integrate the bridging ability of all mobile devices when setting up a partial or complete WCN. The possible techniques considered are either to impose the middleware or virtualization of the Wi-Fi interface on top of the hardware device. The details of the possible techniques will be 
presented in our future publications. The motivation and idea of our research came from the literature.

\section{Expecting outcomes from setting up a WCN}

With the realization of a novel bridging technique, the working environment of a WCN with full connectivity is illustrated in Figure 4. With reference to Figure 4, the highlights are as follows:

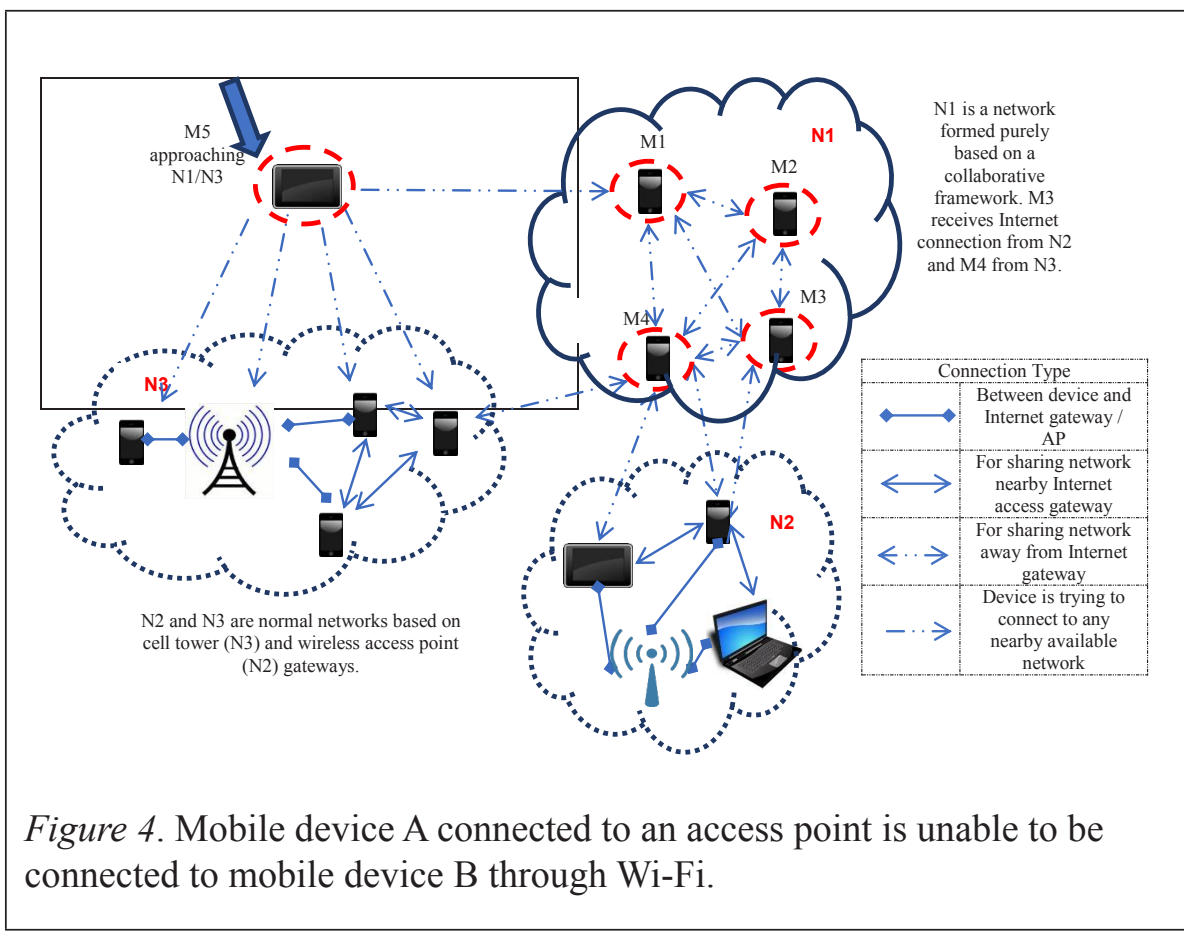

- The directions of all the devices are uncertain.

- $\quad$ Each device in the environment is assumed to be equipped with the functionality to set up a WCN.

- $\quad$ M1 to M5 are mobile devices (Smartphone, Tablet or Laptop) seeking Internet access.

- $\quad$ M5 is moving towards a sharing wireless network nearby and the agent is calculating the best mapping wireless network for Internet connectivity.

- $\quad \mathrm{N} 1$ is a WCN which is set up purely based on mobile agents of M1 to M4 without connectivity to a cell tower or wireless access point.

- $\quad$ N1 Internet connectivity is based on M3 which is derived from one of the mobile devices from N2, and M4 which is derived from one of the mobile devices from N3. 
- $\quad \mathrm{N} 2$ is a WCN established with Internet connectivity based on a wireless access point.

- $\quad \mathrm{N} 3$ is a WCN established with Internet connectivity based on a telecommunication cell tower.

\section{Simulation Tools}

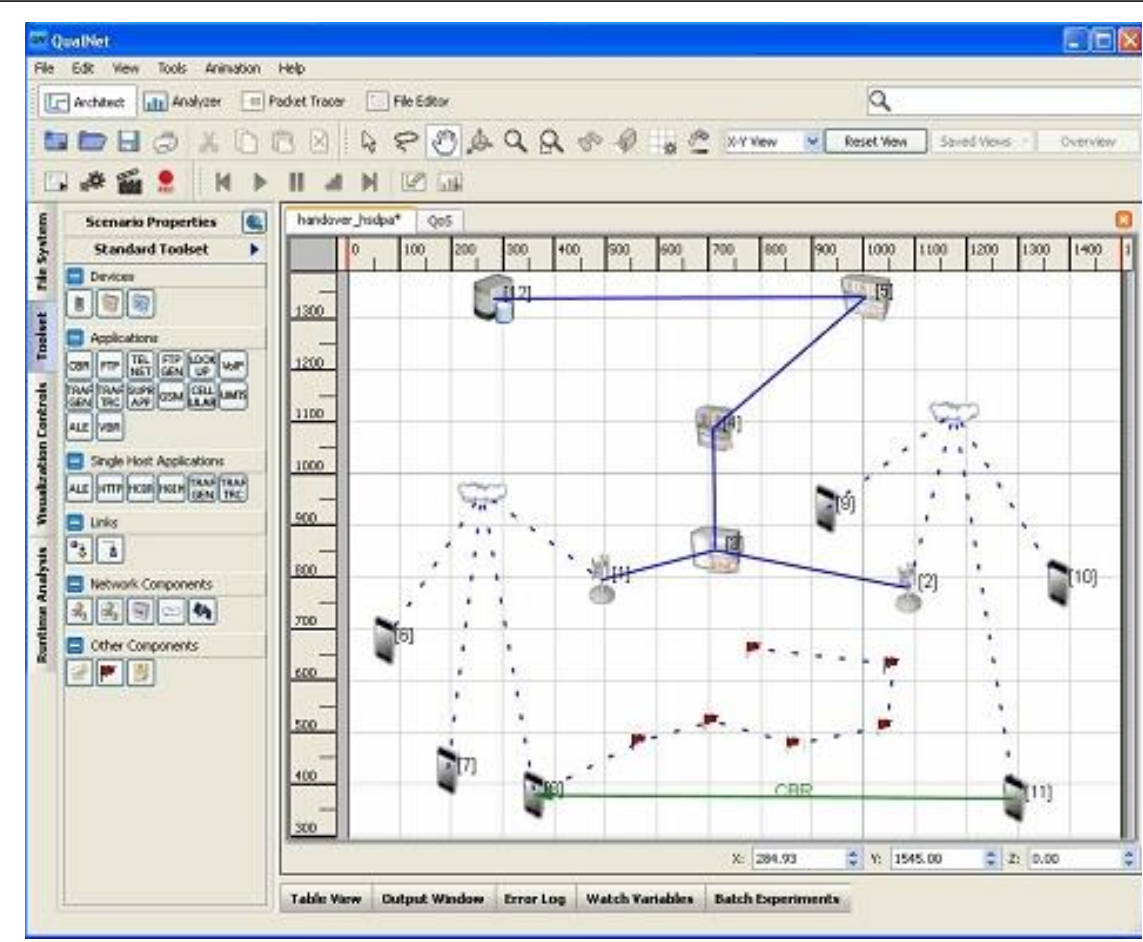

Figure 5. Qualnet simulator to mimic the real environment.

In this section, we suggest some simulation tools which can be used to mimic the behaviour of a real network before the framework or architecture is implemented in the real environment. Qualnet is one of the many well-known network simulators with GUI to visualize real networking environment. Figure 5 shows the user interface of Qualnet which allows the user to perform network scenario-based testing or to test the prototype of the protocol developed before it is implemented in the real environment. The tool also provides a statistical graphing tool for network performance analysis, as in Figure 6. Other than Qualnet, the Network Simulator (NS, NS1 or NS2) is another highly recommended discrete event network simulator targeted on the networking research community. The tool provides flexibility and abstractions 
for the researcher to modify and set up the simulation environment. There are a few parameters involved in order to analyze and measure network connectivity performance. For example, the distance between nodes in metres (or any suitable measurement unit), the throughput or goodput of data transfer rate in bits per second, the wireless signal strength in decibels and the number of hop count in a transfer path. The common metrics are listed in Table 3.

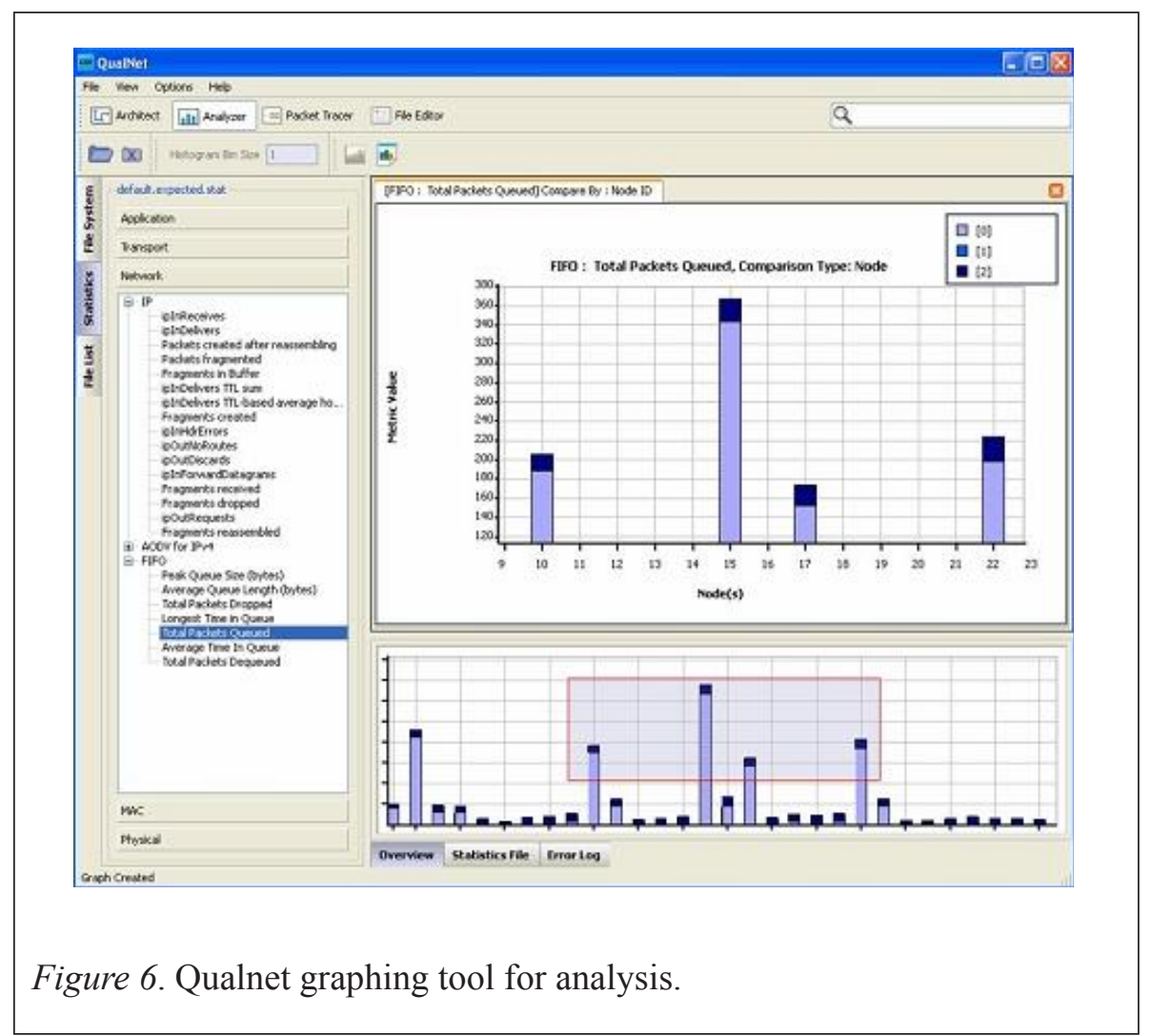

Table 3

The common network metrics for analysis and evaluation phase.

\begin{tabular}{ll}
\hline Parameter & Unit \\
\hline Distance & metre or longer \\
Throughput & kilobits per second or higher \\
Goodput & kilobits per second or higher \\
Signal strength & decibels \\
Number of hop & usually labelled as n \\
\hline
\end{tabular}




\section{The Quality, Stability, and Performance of WCN}

In terms of stability, quality and performance measurement of a network, there are a few approaches introduced by other researchers. Ibrahim (2018) suggested measuring the Level-of-Resilience (LoR) to identify the ability of a network to withstand adverse event. The criteria to measure LoR includes Level-of-Stability-Reduction (LoSR), Level-of-Performance-Reduction (LoPR) and network Recovery-Time (RT). The LoSR is measured by examining the Ip traffic's percentage dropped. The LoPR is measured based on the percentage of reduction on Quality-of-Service (QoS) latency. The RT is measured through the convergence time. Ayisat, Zulkhairi and Azman (2018) has proposed a framework which utilizes the big data collected in a network to model the Quality of Experience (QoE) of the end user. The framework consists of three different phases. The collection phase to gather data required, the data preparation phase to filter and ensure the data fulfill big data characteristics, and the data modeling phase to model the $\mathrm{QoE}$ through predictive and prescriptive analytic processes.

\section{CONCLUSION}

As elaborated earlier, this paper highlighted six constraints and challenges of setting up a WCN based on a systematic review of related research in mobile device ad-hoc networking area. The preliminary testing conducted to validate the constraints and challenges identified was also reported. It was confirmed that the main challenge of setting up WCN was the ability of mobile devices to provide the bridging ability. In the discussion section, the expected outcome of the network architecture upon realization of the bridging functionality was illustrated. However, illustrations of the ideal outcome were based on theoretical and logical concepts which require thorough research and exploration work in the relevant area. The measurement methodology and the relevant metrics for the testing and evaluation stage were described at the end of the article. The focus of future work is to establish bridging functionality. If this main bridging problem can be resolved it will be a breakthrough in setting up a decentralized WCN with mobile devices in the near future.

\section{ACKNOWLEDGEMENT}

This research received no specific grant from any funding agency in the public, commercial, or not-for profit sectors. 


\section{REFERENCES}

Accenture. (2012). Mobile Web Watch Internet Usage Survey. Retrieved from http://www.accenture.com/sitecollectiondocuments/pdf/accenturemobile-web-watch-internet-usage-survey-2012.pdf

Adeyeye, M., \& Gardner-Stephen, P. (2011). The village telco project: A reliable and practical wireless mesh telephony infrastructure. EURASIP Journal on Wireless Communications and Networking, 2011(1), 78. doi.org/10.1186/1687-1499-2011-78

Ayisat, W. Y., Zulkhairi, Md. D., \& Azman, T. (2018). Framework for modelling mobile network quality of experience through big data analytics approach. Journal of Information and Communication Technology, 17(1), 79-113.

Bellavista, P. (2014). Resource sharing in mobile environments: The next frontier of social networking novel middleware for green exploitation of social sharing in opportunistic mobile environments, 2-3.

Bellavista, P., Corradi, A., \& Giannelli, C. (2010). The real ad-hoc multihop peer-to-peer (RAMP) middleware: An easy-to-use support for spontaneous networking. Proceedings - IEEE Symposium on Computers and Communications, 463-470. doi.org/10.1109/ISCC.2010.5546785

Bellavista, P., \& Giannelli, C. (2009). Middleware solutions for selforganizing multi-hop multi-path internet connectivity based on Bluetooth. Lecture Notes of the Institute for Computer Sciences, SocialInformatics and Telecommunications Engineering, 7 LNICST, 58-71. doi.org/10.1007/978-3-642-01802-2_5

Biswas, J., \& Veloso, M. (2010). WiFi localization and navigation for autonomous indoor mobile robots. Proceedings - IEEE International Conference on Robotics and Automation, 4379-4384. doi.org/10.1109/ ROBOT.2010.5509842

Camp, J. D., \& Knightly, E. W. (2008). The IEEE 802.11s extended service set mesh networking standard. IEEE Communications Magazine, 46(8), 120-126. doi.org/10.1109/MCOM.2008.4597114

ExactTarget. (2014). 2014 Mobile behavior report: Combining mobile device tracking and consumer survey data to build a powerful mobile strategy, $1-36$.

Gardner-Stephen, P., Challans, R., Lakeman, J., Bettison, A., GardnerStephen, D., \& Lloyd, M. (2013). The serval mesh: A platform for resilient communications in disaster \& crisis. Proceedings of the $3 \mathrm{rd}$ IEEE Global Humanitarian Technology Conference, GHTC 2013, 162166. doi.org/10.1109/GHTC.2013.6713674

Gardner-Stephen, P., \& Palaniswamy, S. (2011). Serval mesh softwareWiFi multi-model management. Proceedings of the 1st International 
Conference on Wireless Technologies for Humanitarian Relief - ACWR '11, (January 2011), 71. http://doi.org/10.1145/2185216.2185245

Grossglauser, M., \& Tse, D. N. C. (2002). Mobility Increase the Capacity of Ad Hoc Wireless Networks. IEEE/ACM Trans. Netw., 10(4), 477-486. Retrieved from citeseer.ist.psu.edu/grossglauser01mobility.html

Han, H., Liu, Y., Shen, G., \& Member, S. (2014). Design, Realization, and Evaluation of DozyAP for Power-Efficient Wi-Fi Tethering.

Honda, T., \& Ikeda, M. (2013). Improvement of wireless LAN connectivity by optimizing placement of wireless access points. In Information Technology Convergence Security, Robotics, Automations and Communication (Vol. 253). doi.org/10.1007/978-94-007-6996-0

Ibrahim, M. W. (2018). Level of resilience measure for communication networks. Journal of Information and Communication Technology, 17(1), 115-139

Liemhetcharat, S., \& Veloso, M. (2012). Multi-robot map-merging-free connectivity-based positioning and tethering in unknown environments. Progress in Artificial Intelligence, 1(1), 3-23. doi.org/10.1007/s13748011-0007-1

Liu, K., Shen, W., Yin, B., Cao, X., Cai, L. X., \& Cheng, Y. (2016). Development of mobile Ad-hoc networks over Wi-Fi direct with off-the-shelf Android phones. 2016 IEEE International Conference on Communications, ICC 2016. doi.org/10.1109/ICC.2016.7511190

Nungu, A., Brown, T., \& Pehrson, B. (2011). Challenges in sustaining municipal broadband networks in the developing world. In e-Technologies and Networks for Development (pp. 26-40). Springer. doi.org/10.1007/9783-642-22729-5_3

Ocana, M., Bergasa, L. M., Sotelo, M. A., Nuevo, J., \& Flores, R. (2005). Indoor robot localization system using WiFi signal measure and minimizing calibration effort. IEEE International Symposium on Industrial Electronics, IV, 1545-1550. doi.org/10.1109/ISIE.2005.1529162

Osman, M. A., Talib, A. Z., \& Sanusi, Z. A. (2012). A study of the trend of smartphone and its usage behavior in Malaysia, 2(1), 275-286. doi. org/10.1007/978-3-642-22603-8_35

Pries, R., Wendel, V., Staehle, B., Staehle, D., \& Darmstadt, T. U. (2010). Genetic algorithms for wireless mesh network planning. $A C M$ International Conference on Modeling, Analysis, and Simulation of Wireless and Mobile Systems, (October), 226-234.

Reich, J., Misra, V., Rubenstein, D., \& Zussman, G. (2008). Spreadable Connected Autonomic Networks ( SCAN ) Technical Report CUCS016-08.

Sit, T. C. H., Liu, Z., Ang, M. H., \& Seah, W. K. G. (2007). Multi-robot mobility enhanced hop-count based localization in ad hoc networks. 